\title{
CLOSED LINEAR OPERATORS WITH DOMAIN CONTAINING THEIR RANGE
}

\author{
by SCHÔICHI ÔTA
}

(Received 8th February 1984)

\section{Introduction}

In connection with algebras of unbounded operators, Lassner showed in [4] that, if $T$ is a densely defined, closed linear operator in a Hilbert space such that its domain is contained in the domain of its adjoint $T^{*}$ and is globally invariant under $T$ and $T^{*}$, then $T$ is bounded. In the case of a Banach space (in particular, a $C^{*}$-algebra) we showed in [6] that a densely defined closed derivation in a $C^{*}$-algebra with domain containing its range is automatically bounded (see the references in [6] and [7] for the theory of derivations in $C^{*}$-algebras).

In general there exists a densely defined, unbounded closed linear operator with domain containing its range (see Example 3.1). Therefore it is of great interest to study the boundedness and properties of such an operator.

We show in Section 2 that a dissipative closed linear operator in a Banach space with domain containing its range is automatically bounded.

In Section 3, we deal with a densely defined, closed linear operator in a Hilbert space. Using the result in Section 2, we first show that a closed operator which maps its domain into the domain of its adjoint is bounded and, as a corollary, that a closed symmetric operator with domain containing its range is automatically bounded. Furthermore we study some properties of an unbounded closed operator with domain containing its range and show that the numerical range of such an operator is the whole complex plane.

\section{Dissipative operators in Banach space}

Let $\mathscr{X}$ be a Banach space with dual space $\mathscr{X}^{*}$. For $a \in \mathscr{X}$, we denote by $\mathscr{X}^{*}(a)$ the set of all tangent functionals at $a$; that is, $\mathscr{X}^{*}(a)=\left\{\phi \in \mathscr{X}^{*}:\langle a, \phi\rangle=\|a\|\|\phi\|\right\}$.

Let $T$ be a densely defined (linear) operator in $\mathscr{X}$. In this paper, we assume that all operators are linear. We denote by $\mathscr{D}(T)$ the domain of $T$. If, for each $a \in \mathscr{D}(T)$, there exists a non-zero $\phi \in \mathscr{X}^{*}(a)$ such that $\operatorname{Re}\langle T a, \phi\rangle \leqq 0$ then $T$ is called dissipative.

It is well-known [5] that if $T$ is dissipative then $T$ is closable and its closure is also dissipative. Batty recently showed in [1] that if $T$ is dissipative then for all $a \in \mathscr{D}(T)$ and all $\phi \in \mathscr{X}^{*}(a) \quad \operatorname{Re}\langle T a, \phi\rangle \leqq 0$.

Theorem 2.1. Let $T$ be a densely defined, dissipative closed operator in a Banach space $\mathscr{X}$. If there is a positive integer $n$ such that $\mathscr{D}\left(T^{n}\right)$ is dense in $\mathscr{X}$ and is globally invariant under $T$, then $T$ is bounded on $X$.

In particular, if the range of $T$ is contained in $\mathscr{D}(T)$ then $T$ is automatically bounded. 
Proof. For each non-negative integer $m$, we define a norm on $\mathscr{D}\left(T^{m}\right)$ by

$$
|a|_{m}=\sum_{0 \leqq k \leqq m}\left\|T^{k}(a)\right\|
$$

for $a \in \mathscr{D}\left(T^{m}\right)$. Here $T^{0}$ is the identity operator $I$ and ||$_{0}=\|\|$. Since $T$ is closed it follows that $\mathscr{D}\left(T^{m}\right)$ equipped with ||$_{m}$ turns out to be a Banach space, which is denoted by $\left(\mathscr{D}\left(T^{m}\right),||_{m}\right)$.

It is clear that $T$ is also closed as an operator on $\left(\mathscr{D}\left(T^{n}\right), \mid \ln _{n}\right)$ by our assumption. Therefore $T$ is continuous on $\left(\mathscr{D}\left(T^{n}\right),||_{n}\right)$ by the closed graph theorem.

Since $T$ is dissipative, we have, by standard computations,

$$
\|\lambda a-T a\| \geqq \lambda\|a\|
$$

for all $\lambda>0$ and $a \in \mathscr{D}(T)$, so that

$$
|\lambda a-T a|_{n} \geqq \lambda|a|_{n}
$$

for all $\lambda>0, a \in \mathscr{D}\left(T^{n}\right)$. It follows from the semi-group theory that the restriction of $T$ on $\mathscr{D}\left(T^{n}\right)$ generates the uniformly continuous one-parameter semi-group of contractions on $\left(\mathscr{D}\left(T^{n}\right),||_{n}\right)$, and therefore

$$
(I-T) \mathscr{D}\left(T^{n}\right)=\mathscr{D}\left(T^{n}\right)
$$

We show that $\mathscr{D}\left(T^{n}\right)$ is dense in $\left(\mathscr{D}\left(T^{n-1}\right),||_{n-1}\right)$. To see this, let $x$ be in $\mathscr{D}\left(T^{n-1}\right)$. Since $\mathscr{D}\left(T^{n}\right)$ is dense in $\mathscr{X}$ and $(I-T) \mathscr{D}\left(T^{n}\right)=\mathscr{D}\left(T^{n}\right)$, there is a sequence $\left\{x_{l}\right\}$ in $\mathscr{D}\left(T^{n}\right)$ such that $(I-T)^{n-1} x_{l} \rightarrow(I-T)^{n-1} x$ as $l \rightarrow \infty$. By using the inequality (\#), we get elements $y^{(k)}(0 \leqq k \leqq n-1)$ such that

$$
(I-T)^{k} x_{l} \rightarrow(I-T)^{k} y^{(k)} \quad \text { as } \quad l \rightarrow \infty
$$

and

$$
y^{(0)}=x
$$

Since $T$ is closed it follows that $y^{(k)}=T^{k} x(0 \leqq k \leqq n-1)$. Thus $x_{l} \rightarrow x$ in $\left(\mathscr{D}\left(T^{n-1}\right),||_{n-1}\right)$. We next show that $(I-T) \mathscr{D}\left(T^{n}\right)$ is closed in $\left(\mathscr{D}\left(T^{n-1}\right),||_{n-1}\right)$. Take a sequence $\left\{x_{l}\right\}$ in $\mathscr{D}\left(T^{n}\right)$ and $x$ in $\mathscr{D}\left(T^{n-1}\right)$ satisfying $(I-T) x_{l} \rightarrow x$ as $n \rightarrow \infty$ with respect to ||$_{n-1}$. It follows from the inequality ||$_{n-1} \geqq\|\|$ and the dissipativeness of $T$ that there is an element $y$ in $\mathscr{X}$ such that $x_{l} \rightarrow y$ as $l \rightarrow \infty$, so that $T x_{l} \rightarrow y-x$. Since $T$ is closed, it follows that $y$ belongs to $\mathscr{D}(T)$ and $(I-T) y=x$. This means that $y$ belongs to $\mathscr{D}\left(T^{n}\right)$ and $x$ belongs to $(I-T) \mathscr{D}\left(T^{n}\right)=\mathscr{D}\left(T^{n}\right)$. Thus $\mathscr{D}\left(T^{n}\right)=(I-T) \mathscr{D}\left(T^{n}\right)=\mathscr{D}\left(T^{n-1}\right)$, and hence $(I-T) \mathscr{D}\left(T^{n-1}\right)=\mathscr{D}\left(T^{n-1}\right)$.

By repeating the above arguments, we obtain

$$
\mathscr{D}(T)=(I-T) \mathscr{D}(T)=\mathscr{D}\left(T^{0}\right)=\mathscr{X} .
$$


Since $T$ is closed it follows that $T$ is bounded on $\mathscr{X}$. This completes the proof of the theorem.

\section{Operators in Hilbert space}

We first give an example of a densely defined, unbounded closed operator such that its range is contained in its domain.

Example 3.1. Let $\mathscr{X}=l^{2}$ be the Hilbert space of sequences $\{x(n)\}_{n=1,2, \ldots}$ for which $\sum_{n=1}^{\infty}|x(n)|^{2}<+\infty$. Define a subspace $\mathscr{D}$ of $l^{2}$ by

$$
\mathscr{D}=\left\{x=\{x(n)\} \in l^{2} ; \sum_{n=1}^{\infty} n^{2}|x(2 n)|^{2}<+\infty\right\} .
$$

We introduce an operator with domain $\mathscr{D}$ as follows;

$$
T x=(x(2), 0,2 x(4), 0, \ldots, n x(2 n), 0, \ldots
$$

for $x \in \mathscr{D}$, that is;

$$
(T x)(2 n-1)=n x(2 n)
$$

and

$$
(T x)(2 n)=0
$$

for $n=1,2, \ldots$.

Then $T$ is a densely defined operator in $l^{2}$. Since

$$
\left\|T u^{(2 i)}\right\|=i
$$

for unit vectors $u^{(2 i)}=(0,0, \ldots, 0,1,0,0 \ldots), T$ is unbounded. It is easily seen by direct computations that $T$ is closed and leaves its domain invariant (in particular $T^{2}=0$ ). We remark that the numerical range of $T$ coincides with the whole complex place $\mathbb{C}$, as cited below.

Let $T$ be a densely defined operator in a Hilbert space. We put

$$
\mathscr{W}(T)=\{(T x, x) ; x \in \mathscr{D}(T) \text { with }\|x\|=1\}
$$

The set $\mathscr{W}(T)$ is said to be the numerical range of $T$. It is well-known that $\mathscr{W}(T)$ is a convex set in $\mathbb{C}$ and if $\mathscr{W}(T) \neq \mathbb{C}$ then $T$ is closable (see [3] and [8]). Furthermore if $T$ is symmetric, $\mathscr{W}(T)$ lies on the real axis. There exists a (closed) symmetric operator such that $\mathscr{W}(T)$ equals the entire axis of reals (see [8] for its construction). 
Proposition 3.2. Let $T$ be a densely defined, closed operator in a Hilbert space. Suppose the range of $T$ is contained in its domain. If $T$ is unbounded, then the numerical range $\mathscr{W}(T)$ is the whole complex plane $\mathbb{C}$.

Proof. If $\mathscr{W}(T) \neq \mathbb{C}$, then there is constants $\alpha \neq 0$ and $\beta$ in $\mathbb{C}$ such that

$$
\begin{aligned}
\mathscr{W}(\alpha T+\beta) & =\alpha \mathscr{W}(T)+\beta \\
& \subset\{z \in \mathbb{C} ; \operatorname{Re} z \leqq 0\}
\end{aligned}
$$

Clearly, $\alpha T+\beta$ leaves its domain invariant and is dissipative. Hence, by Theorem 2.1, $\alpha T+\beta$ is bounded; that is, $T$ is bounded. This is a contradiction.

As seen in Example 3.1, a closed operator with range contained in its domain is not always bounded. We next show that some special operator with this range property is bounded. To see this, we present the following theorem.

Theorem 3.3. Let $T$ be a densely defined, closed operator in a Hilbert space. Suppose $T$ maps its domain into the domain $\mathscr{D}\left(T^{*}\right)$ of its adjoint $T^{*}$. Then $T$ is bounded.

Proof. Let $T=V|T|$ be its polar decomposition. Our assumption implies that

$$
\mathscr{D}\left(T^{*} T\right)=\mathscr{D}(T)=\mathscr{D}(|T|)
$$

Hence $|T|$ leaves its domain invariant. It follows from Theorem 2.1 that $|T|$ is bounded and so $T$ is bounded.

Corollary 3.4. Let $T$ be a densely defined, closed symmetric operator in a Hilbert space. Suppose the domain $\mathscr{D}(T)$ is globally invariant under $T$. Then $T$ is bounded.

Let $T$ be a densely defined, closed operator in a Hilbert space. Define the scalar product on $\mathscr{D}(T)$ by

$$
(x, y)_{T}=(x, y)+(T x, T y)
$$

for $x, y \in \mathscr{D}(T)$. Then $\mathscr{D}(T)$ becomes a Hilbert space, which is denoted by $\left(\mathscr{D}(T),(,)_{T}\right)$. We denote by $\mathscr{R}(T)$ the range of $T$.

Proposition 3.5. Let $T$ be a densely defined, closed linear operator in a Hilbert space $\mathscr{H}$. If the domain $\mathscr{D}(T)$ of $T$ is globally invariant under $T$; that is, $\mathscr{R}(T) \subset \mathscr{D}(T)$, then the intersection $\mathscr{D}(T) \cap \mathscr{D}\left(T^{*}\right)$ is a core for $T$.

Proof. Suppose $T$ satisfies the condition. By the same argument as in the proof of Theorem 2.1, one can choose a real constant $\mu$ (greater than the norm of $T$ as an operator on $\left.\left(\mathscr{D}(T),(,)_{T}\right)\right)$ such that $\mu-T$ is an invertible operator with range equal to 
$\mathscr{D}(T)$. Put $k=\mu-T$. Then $K \mathscr{D}(K)=\mathscr{D}(K)$. Since $|K|$ is self-adjoint, $\mathscr{D}\left(|K|^{2}\right)$ is a core for $|K|$. Therefore, $\mathscr{D}\left(K^{*} K\right)=\mathscr{D}\left(|K|^{2}\right)$ is a core for $K$. It follows that

$$
\begin{aligned}
\mathscr{D}(T) & =K \mathscr{D}(T) \\
& \leqq \overline{K \mathscr{D}\left(K^{*} K\right)^{K}} \\
& \subseteq \overline{\mathscr{D}(K) \cap \mathscr{D}\left(K^{*}\right)^{K}} \\
& =\overline{\mathscr{D}(T) \cap \mathscr{D}\left(T^{*}\right)^{K}}
\end{aligned}
$$

where ${ }^{-}{ }^{K}$ denotes the closure in the Hilbert space $\left(\mathscr{D}(K)=\mathscr{D}(T),(,)_{K}\right)$ induced by $K$. Since the above topologies induced by $T$ and $K$ on $\mathscr{D}(T)$ coincide, it follows that $\mathscr{D}(T) \cap \mathscr{D}\left(T^{*}\right)$ is a core for $T$.

\section{REFERENCES}

1. C. J. K. BAtTY, Dissipative mappings and well-behaved derivations, J. London Math. Soc (2), 18 (1978), 527-533.

2. E. B. Davies, One-parameter semi-groups (New York-San Francisco-London, Academic Press, 1980).

3. T. Kato, Perturbation theory for linear operators (Berlin-Heidelberg-New York, Springer, 1966).

4. G. LASSNER, Topological algebras of operators, Rep. Math. Phys. 3 (1972), 279-293.

5. G. Lummer and R. S. Phillips, Dissipative operators in a Banach space, Pacific J. Math. 11 (1961), 679-689.

6. S. ÔTA, Closed derivations in $C^{*}$-algebras, Math. Ann. 257 (1981), 239-250.

7. S. ÔTA, Commutants of unbounded derivations in $C^{*}$-algebras, J. Reine Angew. Math. 347 (1984), 21-32.

8. M. H. STONE, Linear transformations in Hilbert space and their applications to analysis (Amer. Math. Soc. Colloq. Publ. 15, Providence, R.I., 1932).

Department of Mathematics

KYUSHU UNIVERSITY 33

FUKUOKA 812 , JAPAN 